Review Article

\title{
Coronavirus Disease 2019-Related Multisystem Inflammatory Syndrome in Children: A Systematic Review and Meta-Analysis
}

\author{
Ji-Gan Wang $\mathbb{D},{ }^{1}$ Zhi-Juan Zhong, ${ }^{2}$ Meng Li, ${ }^{1}$ Jun Fu, ${ }^{1}$ Yu-Heng Su, ${ }^{1}$ You-Min Ping, \\ Zi-Ji Xu, ${ }^{1}$ Hao Li, ${ }^{1}$ Yan-Hao Chen, ${ }^{1}$ and Yu-Li Huang ${ }^{1}$ \\ ${ }^{1}$ Pediatrics Department, Maternal and Child Health Hospital of Guangxi Zhuang Autonomous Region, Nanning, China \\ ${ }^{2}$ Pediatrics Department, Hainan General Hospital, Hainan Affiliated Hospital of Hainan Medical University, Haikou, China
}

Correspondence should be addressed to Ji-Gan Wang; 354713144@qq.com

Received 2 February 2021; Revised 17 May 2021; Accepted 10 July 2021; Published 23 July 2021

Academic Editor: Danni Zheng

Copyright (C) 2021 Ji-Gan Wang et al. This is an open access article distributed under the Creative Commons Attribution License, which permits unrestricted use, distribution, and reproduction in any medium, provided the original work is properly cited.

Background. This study aimed to describe the clinical symptoms, laboratory findings, treatment, and outcomes of coronavirus disease 2019-related multisystem inflammatory syndrome in children to provide a reference for clinical practice. Methods. We employed a literature search of databases such as PubMed, Web of Science, EMBASE, and Johns Hopkins University for articles on COVID-19-related multisystem inflammatory syndrome in children published between April 1, 2020, and January 15, 2021. High-quality articles were selected for analysis on the basis of their quality standard scores. Using R3.6.3 software, meta-analyses of random- or fixed-effects models were used to determine the prevalence of comorbidities. Subgroup analysis was also performed to determine heterogeneity. Results. A total of 57 articles (2,290 pediatric patients) were included in the study. Clinical Manifestations. :ncidences of fever, gastrointestinal symptoms, respiratory symptoms, and musculoskeletal symptoms (myalgias or arthralgias) were 99.91\% (95\% CI: 99.67-100\%), 82.72\% (95\% CI: 78.19-86.81\%), 53.02\% (45.28-60.68\%), and 14.16\% (95\% CI: 8.4-21.12\%), respectively. The incidences of rash, conjunctival injection, lymphadenopathy, dry cracked lips, neurologic symptoms (headache, altered mental status, or confusion), swollen hands and feet, typical Kawasaki disease, and atypical Kawasaki disease were 59.34\% (95\% CI: 54.73-63.87\%), 55.23\% (95\% CI: 50.22-60.19\%), 27.07\% (95\% CI: 19.87-34.93\%), 46.37\% (95\% CI: 39.97-52.83\%), 28.87\% (95\% CI: 22.76-35.40\%), 28.75\% (95\% CI: 21.46-36.64\%), $17.32 \%$ (95\% CI: $15.44-19.29 \%)$, and 36.19\% (95\% CI: $21.90-51.86 \%$ ), respectively. The incidences of coronary artery dilation, aneurysm, pericardial effusion, myocarditis, myocardial dysfunction, high troponin, and $N$-terminal pro-B-type natriuretic peptide were $17.83 \%, 6.85 \%, 20.97 \%, 35.97 \%$, $56.32 \%, 76.34 \%$, and $86.65 \%$, respectively. The incidences of reduced lymphocytes, thrombocytopenia, hypoalbuminemia, elevated C-reactive protein, ferritin, LDH, interleukin-6, PCT, and FIB were 61.51\%, 26.42\%, 77.92\%, 98.5\%, 86.79\%, 80.59\%, 89.30\%, 85.10\%, and 87.01\%, respectively. PICU Hospitalization Rate and Mortality. The incidences of PICU hospitalization or with shock were $72.79 \%$ and $55.68 \%$, respectively. The mortality rate was $1.00 \%$. Conclusion and Relevance. PICU hospitalization and shock rates of multisystem inflammatory syndrome in children associated with COVID-19 were high, and its cumulative multiorgans and inflammatory indicators are increased, but if treated in time, the mortality rate was low.

\section{Introduction}

The transmission of coronavirus disease 2019 (COVID-19) was recognized by the World Health Organization as a global pandemic in March 2020 [1]. In the early stages of the pandemic, it was widely believed that children were not susceptible to infection and the disease was benign disease [2]. However, as the pandemic progressed and children died, people began to pay attention to this part of the population
[3]. In April 2020, eight children were reported in the United Kingdom with hyperinflammatory shock, showing features similar to atypical Kawasaki disease-Kawasaki disease shock syndrome [4]. This first report was followed by more in the United States and other regions thereafter [5] and became known as "multisystem inflammatory syndrome in children" (MIS-C). The syndrome was clinically similar to Kawasaki disease (KD), as well as toxic shock syndrome and secondary lymphoid tissue cells hyperplasia/macrophage 
activation syndrome [6]. Since little was known about this new syndrome, people were extremely worried due to the severity of the condition. Therefore, a systematic description of the clinical manifestations, laboratory tests, and prognosis of this disease is necessary.

\section{Materials and Methods}

2.1. Study Registration. The study is reported in accordance with the 2009 guidelines of the Preferred Reporting Items for Systematic Reviews and Meta-Analyses (PRISMA) statement (Supplementary Materials) [7]. This systematic review and meta-analysis is registered in the Prospero International Prospective Register of Systematic Reviews (CRD42020208680).

2.2. Definition Criteria. As the disease was discovered in April as a new disease, the name and definition of the disease during the first 2 months were quite different. Therefore, "pediatric inflammatory multisystem syndrome temporally associated with SARS-CoV-2 pandemic (PIMS-TS)" and "multisystem inflammatory syndrome in children (MIS-C)" and "SARS-CoV-2-induced Kawasaki-like hyperinflammatory syndrome (SCiKH syndrome)" were included in the analysis [8].

2.3. Computer Retrieval of Articles. Searches for publications reporting on MIS-C associated with COVID-19 in PubMed, Web of Science, Embase, and Johns Hopkins University published data were conducted. The retrieval time for data was from April 1, 2020, to January 15, 2021. At the same time, online database and manual retrievals were conducted, and the references included in the articles were also traced. Subjects and free words were used in the retrieval, and adjustments were made according to the characteristics of different databases. Data retrieval was not limited to any language or country. For the PubMed search strategy, three search categories were used in combination (\#1 AND \#2 AND \#3) as follows:

\#1 (children) OR (child)) OR (kid) OR (pediatric) OR
(paediatric)
\#2 (multisystem inflammatory syndrome) OR (MIS-C)
OR (PIMS-TS) OR (SCiKH syndrome) OR (SARS-
CoV-2-induced Kawasaki-like hyperinflammatory
syndrome) OR (Kawasaki)
\#3 (2019-nCoV) OR (COVID-19) OR (SARS-CoV-2)
OR (Corona Virus Disease 2019) OR (coronavirus)

2.4. Literature Screening and Data Extraction. Two researchers (J. G. W. and Z. J. Z.) independently searched and screened articles as well as collected and cross-checked data. If there was any dispute, it was discussed or negotiated with a third researcher (M. L.).

Inclusion criteria were (1) study type: cohort studies, case-control studies, case series, and cross-sectional studies; (2) participants: (<21 years old) with MIS-C; and (3) observation index: clinical manifestations (including fever and cough), laboratory examination, severe cases, and deaths.

Exclusion criteria were (1) adult cases; (2) case reports; and (3) incomplete, missing, or inaccessible data.

The level of laboratory test items was determined according to the following standards (reference ranges were obtained from Nelson Textbook of Pediatrics): normal white blood cell: 5.5 to $12.0 \times 109 / \mathrm{L}$, leukocytosis: more than $12.0 \times 109 / \mathrm{L}$, leukopenia: less than $5.5 \times 109 / \mathrm{L}$, lymphopenia: less than $1.2 \times 109 / \mathrm{L}$, high PCT: more than $0.046 \mathrm{ng} / \mathrm{mL}$, high CRP: more than $10 \mathrm{mg} / \mathrm{L}$, high LDH: more than $300 \mathrm{U} /$ L, high ALT: more than $45 \mathrm{U} / \mathrm{L}$, high AST: more than $50 \mathrm{U} /$ L, high creatinine: more than $62 \mu \mathrm{mol} / \mathrm{L}$, high blood urea nitrogen: more than $7.1 \mathrm{mmol} / \mathrm{L}$, high $\mathrm{CK}$ : more than $170 \mathrm{U} /$ L, high CK-MB: more than $25 \mathrm{U} / \mathrm{L}$, high D-dimer: more than $0.55 \mathrm{mg} / \mathrm{L}$, high ferritin: more than $150 \mathrm{ng} / \mathrm{mL}$, high interleukin-6: more than $5 \mathrm{pg} / \mathrm{mL}$, and high $N$-terminal pro-Btype natriuretic peptide: more than $194.0 \mathrm{pg} / \mathrm{ml}$. We excluded studies that did not report original data or clear diagnostic criteria and no relevant outcome.

Diagnostic criteria of coronary artery dilatation (CAD) and coronary artery aneurysms (CAA) were as follows: $Z$ value $<2$ is no $C A D, 2 \leq Z$ value $<2.5$ is $C A D, 2.5 \leq Z$ value $<5$ is small CAA, CAA inner diameter $<0.8 \mathrm{~cm}$ and $5 \leq Z$ value $<10$ is medium CAA, and CAA inner diameter $\geq 0.8 \mathrm{~cm}$ and $Z$ value $\geq 10$ is large or huge CAA.

2.5. Quality Evaluation of the Included Studies. This was a case series study that adhered to the National Institute for Clinical excellence guidelines for quality evaluation [9]. The evaluation items were as follows: (1) cases in the case series came from medical institutions at different levels and from different research centers; (2) the research hypothesis or purpose was clearly described; (3) clear reports were included in the exclusion criteria; (4) measurement results were clearly defined; (5) collected data achieved the expected purpose; (6) the patient recruitment period was clearly defined; (7) the main findings were clearly described; (8) the results were analyzed and reported in layers. One point was awarded for each item (maximum 8 points), with a total score $\geq 4$ indicating high-quality research. Two researchers independently evaluated the quality and cross-checked the results.

2.6. Statistical Analysis. Statistical analyses were conducted using the Meta 4.11-0 Package in R Software Version 3.6.3. Before meta-analysis, the conversion of the original rate to conform to a normal distribution was carried out first. The conversion methods included PRAW (original rate without conversion), PLN (logarithmic conversion), PLOGIT (logit conversion), PAS (arcsine conversion), and PFT (Freeman-Tukey dual arcsine conversion). The meta-analysis was carried out on the normal distribution or mode closest to the state distribution. A random- or fixed-effects model was selected according to heterogeneity: if $P<0.1$ and $I^{2} \leq 50 \%$, a fixed model was used; and if $P<0.1$ and $I^{2}>50 \%$, the study was considered to have heterogeneity and a random-effects 
model was used [10]. According to the sample size of each independent study, different weights were given and the effect rate of each independent sample was combined to calculate the incidence rate and $95 \%$ confidence interval (CI). To explore heterogeneity, we performed subgroup analyses based on the location (region) and sample size $(<50$, $\geq 50$ ). Finally, a funnel graph was created and the publication offset was statistically tested using the Egger method.

2.7. Ethics. As this is a systematic review, ethical approval was not required.

\section{Results}

3.1. Literature Screening Process and Results. A total of 685 related articles were initially retrieved, of these, 331 duplicate articles were deleted and an additional 227 articles were deleted based on a review of the titles and abstract. Finally, after layer-by-layer screening, a total of 57 articles [5, 11-66] were included $(2,290$ children), the majority of which were from Europe and the United States. The male to female ratio was $1.49: 1$. The literature screening process and results are shown in Figure 1. The characteristics of the included studies are shown in Supplementary Table 1.

\subsection{Basic Characteristics and Quality Evaluation Results of the} Included Studies. The quality characteristics of all included studies were $4-8$ points, indicating high-quality studies ( $\geq 4$ points; Supplementary Table 2).

\subsection{Meta-Analysis Results (Table 1)}

3.3.1. Clinical Manifestations. The incidences of fever, gastrointestinal symptoms, and musculoskeletal symptoms (myalgias or arthralgias) were 99.91\% (95\% CI: 99.67-100\%), $82.72 \%$ (95\% CI: $78.19-86.81 \%$ ), and $14.16 \%$ (95\% CI: $8.4-21.12 \%)$, respectively.

The incidences of rash, conjunctival injection, lymphadenopathy, dry cracked lips, neurologic symptoms (headache, altered mental status, or confusion), swollen hands and feet, typical KD, and atypical KD were 59.34\% (95\% CI: 54.73-63.87\%), 55.23\% (95\% CI: 50.22-60.19\%), $27.07 \% \quad(95 \% \quad$ CI: $\quad 19.87-34.93 \%), \quad 46.37 \% \quad(95 \% \quad$ CI: 39.97-52.83\%), 28.87\% (95\% CI: $22.76-35.40 \%), 28.75 \%$ (95\% CI: $21.46-36.64 \%), 17.32 \%$ (95\% CI: $15.44-19.29 \%)$, and $36.19 \%(21.90-51.86 \%)$, respectively.

3.3.2. Cardiac Damage. The incidences of coronary artery dilation, aneurysm, pericardial effusion, myocarditis, cardiac systolic function affects, high troponin, and $\mathrm{N}$-terminal proB-type natriuretic peptide were $18.2 \%$ (95\% CI: 12.0-25.5\%), 6.7\% (95\% CI: $2.8-12.1 \%$ ), 21.5\% (95\% CI:14.3-29.7\%), 36\% (95\% CI: $32.9-39.1 \%), 62.5 \%$ (95\% CI: $51.2-73.3 \%), 79.6 \%$ (95\% CI: $66.7-90.0 \%$ ), and $89.7 \%$ (95\% CI: $79.1-96.7 \%$ ), respectively.
3.3.3. Laboratory Examinations. The incidences of reduced lymphocytes, thrombocytopenia, hypoalbuminemia, elevated white blood cell count, leukopenia, C-reactive protein (CRP), ferritin, $\mathrm{LDH}$, erythrocyte sedimentation rate (ESR), interleukin-6 (IL-6), PCT, and FIB were $61.51 \%$ (95\% CI: $50.73-71.74 \%), 26.42 \%$ (95\% CI: 18.19-35.58\%), $77.92 \%$ (95\% CI: $66.00-87.85 \%$ ), $55.30 \%$ (95\% CI: $40.47-69.66 \%$ ), $4.40 \%$ (95\% CI: $0.14-14.13 \%$ ), 98.5\% (95\% CI: 95.04-99.65\%), 90\% (95\% CI: 80.0-97.0\%), $80.59 \% \quad(95 \% \quad$ CI: $\quad 42.53-99.73 \%), \quad 82.44 \% \quad(95 \% \quad$ CI: 73.47-89.89\%), 89.3\% (95\% CI: 75.3-97.88\%), 85.10\% (95\% CI: $75.65-92.55 \%)$, and $87.01 \%$ (95\% CI: $73.97-95.98 \%)$, respectively.

3.3.4. Organ Injury. The incidences of liver function damage, kidney damage, and lung ground-glass changes were $46.29 \% \quad(95 \%$ CI: $\quad 32.78-60.08 \%), \quad 27.67 \% \quad(95 \%$ CI: $20.83-35.08 \%$ ), and $24.69 \%$ (95\% CI: $19.74-29.99 \%$ ), respectively.

3.3.5. PICU Hospitalization Rate and Mortality. The incidences of PICU hospitalization or with shock were $72.79 \%$ $(95 \%$ CI: $66.75-78.44 \%)$ and $55.68 \% \quad(95 \%$ CI: $48.50-62.74 \%)$, respectively. The mortality rate was $1.00 \%$ (95\% CI: $0.61-1.48 \%)$.

3.3.6. Treatment. The proportions of cases using invasive mechanical ventilation, ECMO, IVIG, aspirin, glucocorticoid, vasoactive agent, positive inotropic, IL-1 receptor antagonist, IL-6 receptor antagonist, or infliximab antibiotic were $22.68 \%$ (95\% CI: 16.91-29.02\%), $0.48 \%$ (95\% CI: 0.03-1.42\%), 82.15\% (95\% CI: 76.53-87.14\%), 67.97\% (95\% CI: $53.77-80.64 \%$ ), 59.32\% (95\% CI: 52.02-66.43\%), 45.79\% (95\% CI: 42.62-48.97\%), 58.99\% (95\% CI:48.57-69.02\%), $3.61 \%$ (95\% CI:0.68-8.73\%), 10.9\% (95\% CI: $2.79-23.42 \%$ ), 6.68\% (95\% CI: $3.03-11.62 \%)$, and $90.96 \%$ (95\% CI: $82.29-96.92 \%)$, respectively.

\subsection{Subgroup Analysis}

3.4.1. Heterogeneity. In this study, except for fever, other factors had obvious heterogeneity $\left(I^{2}, 39-98\right)$. In order to explore the source of heterogeneity, the subjects were classified according to region (United States, Europe, and other countries) and sample size $(<50, \geq 50)$ and grouped by gastrointestinal symptoms, lymphopenia, and respiratory symptoms. The results of subgroup analyses were consistent with the overall results, and there was no significant difference between the heterogeneity of each subgroup and overall heterogeneity, indicating that the region and sample size of the study were not the main source of heterogeneity (Table 2).

3.4.2. Sensitivity Analysis. Sensitivity analysis was conducted for gastrointestinal symptom indicators. After removing each study in turn, the statistics were combined 


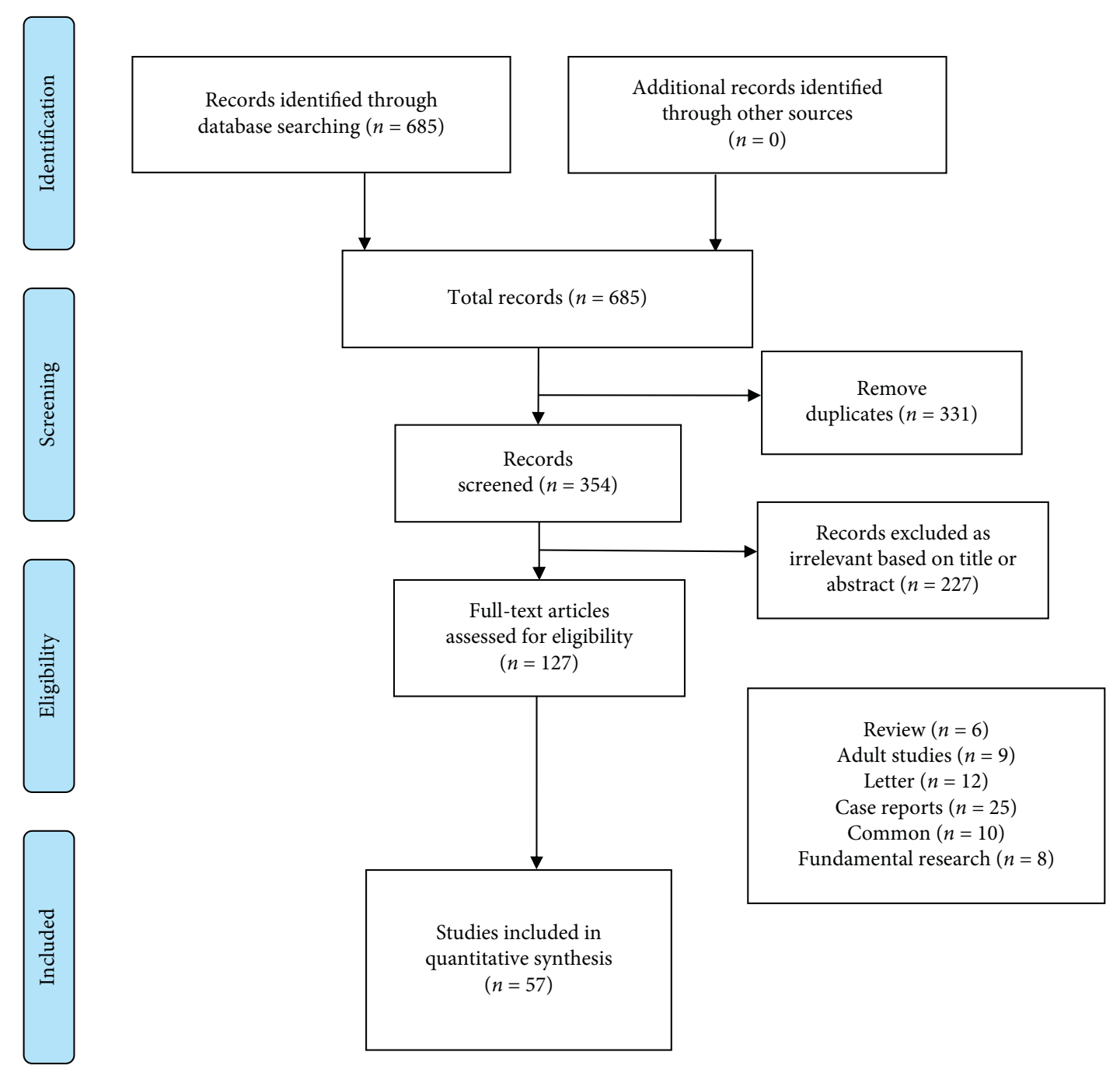

Figure 1: Flow diagram for identification of selected studies in the meta-analysis.

again. The results did not change significantly, indicating that the results were relatively stable (Figure 2).

3.5. Publication Bias. According to the meta-analysis of gastrointestinal symptoms, a funnel plot was drawn. The results showed that there was good symmetry between the left and right distributions of each research point (Figure 3). The Egger test $(P=0.943)$ results suggested that there are no publication biases.

\section{Discussion}

It seems that the nomenclature for multisystem inflammatory syndrome in children has not been unified, with some countries referring to it as "pediatric inflammatory multisystem syndrome temporally associated with SARSCoV-2 pandemic (PIMS-TS)" or "SARS-CoV-2-induced KD-like hyperinflammatory syndrome (SCiKH syndrome)"; however, "multisystem inflammatory syndrome in children (MIS-C)" is the most commonly used term [67]. Although the definition of this disease is still inconsistent, its common characteristics include fever, multiple organ damage, and increased inflammatory indicators. Since April 2020, eight cases of children with high inflammatory shock COVID-19 were reported in London, UK [5], which increased attention on the recently recognized syndrome due to its severe effects, disbanding the previous notion that children with COVID19 presented with a mild disease course. Almost 100\% of these patients had fever, and only one study from Iran [24] reported patients that did not have fever (4/45 patients). In addition to fever, gastrointestinal symptoms $(82.72 \%)$ are the second most common clinical manifestation. Because children are often accompanied by cardiac inflammation and some common features of KD, the term COVID-19related MIS-C was created to describe this novel manifestation and establish diagnostic criteria. The clinical manifestations included repeated high fever, rash, conjunctivitis, peripheral edema, and extensive limb pain, accompanied by obvious gastrointestinal symptoms, which made it difficult to perform volume resuscitation. Finally, norepinephrine and milrinone were needed to provide hemodynamic 
TABLE 1: Meta-analysis results.

\begin{tabular}{|c|c|c|c|c|c|c|c|}
\hline \multirow[b]{2}{*}{ Outcome indicators } & \multirow{2}{*}{$\begin{array}{l}\text { Number of included } \\
\text { studies }\end{array}$} & \multirow{2}{*}{$\begin{array}{l}\text { Sample } \\
\text { size }\end{array}$} & \multicolumn{3}{|c|}{ Heterogeneity } & \multirow{2}{*}{$\begin{array}{l}\text { Effect of } \\
\text { model }\end{array}$} & \multirow{2}{*}{$\begin{array}{c}\text { Meta-analysis results } \\
\text { R\% (95\% CI) }\end{array}$} \\
\hline & & & $\begin{array}{c}P \\
\text { values }\end{array}$ & $I^{2}$ & $t^{2}$ & & \\
\hline \multicolumn{8}{|l|}{ Clinical features } \\
\hline Fever & 47 & 1367 & 0.86 & 0 & 0.71 & Fixed & $99.94 \%(99.74 \%-100 \%)$ \\
\hline Rash & 46 & 1953 & $<0.01$ & $64.30 \%$ & 0.0118 & Random & $\begin{array}{c}59.34 \%(54.73 \%- \\
63.87 \%)\end{array}$ \\
\hline Dry cracked lips & 30 & 1634 & $<0.01$ & $77.10 \%$ & 0.0181 & Random & $\begin{array}{c}46.37 \%(39.97 \%- \\
52.83 \%)\end{array}$ \\
\hline Conjunctival injection & 37 & 1799 & $<0.01$ & $65.70 \%$ & 0.0112 & Random & $\begin{array}{c}55.23 \%(50.22 \%- \\
60.19 \%)\end{array}$ \\
\hline Swollen hands and feet & 24 & 998 & $<0.01$ & $81.30 \%$ & 0.0291 & Random & $\begin{array}{c}28.75 \%(21.46 \%- \\
36.64 \%)\end{array}$ \\
\hline Lymphadenopathy & 21 & 1513 & $<0.01$ & $86.60 \%$ & 0.0267 & Random & $\begin{array}{c}27.07 \%(19.87 \%- \\
34.93 \%)\end{array}$ \\
\hline Gastrointestinal symptoms & 51 & 2126 & $<0.01$ & $80.00 \%$ & 0.0264 & Random & $\begin{array}{c}82.72 \% \\
(78.19 \%-86.81 \%)\end{array}$ \\
\hline Neurological symptoms & 31 & 1668 & $<0.01$ & $82.10 \%$ & 0.0248 & Random & $\begin{array}{c}28.87 \% \\
(22.76 \%-35.40 \%)\end{array}$ \\
\hline Respiratory symptoms & 36 & 1753 & $<0.01$ & $86.85 \%$ & 0.0378 & Random & $\begin{array}{c}53.02 \% \\
(45.28 \%-60.68 \%)\end{array}$ \\
\hline Arthralgias & 9 & 393 & 0.03 & $53.50 \%$ & 0.0084 & Random & $14.16 \%(8.4 \%-21.12 \%)$ \\
\hline Typical Kawasaki disease & 32 & 1481 & $<0.01$ & $92.60 \%$ & 0.0798 & Random & $\begin{array}{c}17.32 \% \\
(15.44 \%-19.29 \%)\end{array}$ \\
\hline Atypical Kawasaki disease & 18 & 559 & $<0.01$ & $91.60 \%$ & 0.0981 & Random & $\begin{array}{c}36.19 \% \\
(21.90 \%-51.86 \%) \\
\end{array}$ \\
\hline \multicolumn{8}{|l|}{ Cardiac damage } \\
\hline Coronary artery dilation & 35 & 927 & $<0.01$ & $64.20 \%$ & 0.0186 & Random & $\begin{array}{c}17.83 \% \\
(13.29 \%-22.87 \%)\end{array}$ \\
\hline Aneurysm & 26 & 734 & $<0.01$ & $66.40 \%$ & 0.0188 & Random & $6.85 \%(3.68 \%-10.92 \%)$ \\
\hline Pericardial effusion & 24 & 1244 & $<0.01$ & $69.30 \%$ & 0.0137 & Random & $\begin{array}{c}20.97 \% \\
(15.69 \%-26.79 \%)\end{array}$ \\
\hline Myocarditis & 20 & 930 & $<0.01$ & $92.10 \%$ & 0.098 & Random & $35.97 \%(32.92 \%-39.08)$ \\
\hline $\begin{array}{l}\text { cardiac systolic function } \\
\text { affects }\end{array}$ & 35 & 1308 & $<0.01$ & $85.70 \%$ & 0.049 & Random & $\begin{array}{c}56.32 \% \\
(47.65 \%-64.80 \%)\end{array}$ \\
\hline High troponin & 30 & 1485 & $<0.01$ & $95.50 \%$ & 0.1324 & Random & $\begin{array}{c}76.34 \% \\
(63.27 \%-87.27 \%)\end{array}$ \\
\hline $\mathrm{BNP}$ & 31 & 1554 & $<0.01$ & $94.20 \%$ & 0.0971 & Random & $\begin{array}{c}84.65 \% \\
(74.95 \%-92.31 \%) \\
\end{array}$ \\
\hline \multicolumn{8}{|l|}{ Organ damage } \\
\hline Liver function damage & 16 & 411 & $<0.01$ & $82.20 \%$ & 0.0524 & Random & $\begin{array}{c}46.29 \% \\
(32.78 \%-60.08 \%)\end{array}$ \\
\hline Renal function & 26 & 1463 & $<0.01$ & $84.90 \%$ & 0.0296 & Random & $\begin{array}{c}27.67 \% \\
(20.83 \%-35.08 \%) \\
\end{array}$ \\
\hline \multicolumn{8}{|l|}{ Laboratory inspection } \\
\hline Leukocytosis & 12 & 401 & $<0.01$ & $73.30 \%$ & 0.0394 & Random & $\begin{array}{c}55.30 \% \\
(40.47 \%-69.66 \%)\end{array}$ \\
\hline Leukopenia & 10 & 417 & $<0.01$ & $82.90 \%$ & 0.0514 & Random & $4.40 \%(0.14 \%-14.13 \%)$ \\
\hline Reduced lymphocytes & 28 & 1206 & $<0.01$ & $89.00 \%$ & 0.0612 & Random & $\begin{array}{c}61.51 \% \\
(50.73 \%-71.74 \%)\end{array}$ \\
\hline Elevated platelets & 11 & 421 & 0.05 & $44.90 \%$ & 0.0093 & Fixed & $\begin{array}{c}10.35 \% \\
(7.62 \%-13.43 \%)\end{array}$ \\
\hline Thrombocytopenia & 20 & 1368 & $<0.01$ & $87.70 \%$ & 0.0326 & Random & $\begin{array}{c}26.42 \% \\
(18.19 \%-35.58 \%\end{array}$ \\
\hline CRP rise & 34 & 1087 & $<0.01$ & $82.20 \%$ & 0.0397 & Random & $\begin{array}{c}98.5 \% \\
(95.04 \%-99.65 \%)\end{array}$ \\
\hline Elevated ferritin & 28 & 884 & $<0.01$ & $84.00 \%$ & 0.0483 & Random & $\begin{array}{c}86.79 \% \\
(79.32 \%-92.79 \%)\end{array}$ \\
\hline Elevated ESR & 13 & 475 & $<0.01$ & $75.90 \%$ & 0.0253 & Random & $\begin{array}{c}82.44 \% \\
(73.47 \%-89.89 \%)\end{array}$ \\
\hline Elevated PCT & 17 & 597 & $<0.01$ & $81.80 \%$ & 0.0414 & Random & $\begin{array}{c}85.10 \% \\
(75.65 \%-92.55 \%) \\
\end{array}$ \\
\hline
\end{tabular}


TABLE 1: Continued.

\begin{tabular}{|c|c|c|c|c|c|c|c|}
\hline \multirow[b]{2}{*}{ Outcome indicators } & \multirow{2}{*}{$\begin{array}{l}\text { Number of included } \\
\text { studies }\end{array}$} & \multirow[b]{2}{*}{$\begin{array}{l}\text { Sample } \\
\text { size }\end{array}$} & \multicolumn{3}{|c|}{ Heterogeneity } & \multirow[b]{2}{*}{$\begin{array}{l}\text { Effect of } \\
\text { model }\end{array}$} & \multirow{2}{*}{$\begin{array}{c}\text { Meta-analysis results } \\
\text { R\% (95\% CI) }\end{array}$} \\
\hline & & & $\begin{array}{c}P \\
\text { values }\end{array}$ & $I^{2}$ & $t^{2}$ & & \\
\hline Elevated FIB & 13 & 414 & $<0.01$ & $86.80 \%$ & 0.0658 & Random & $\begin{array}{c}87.01 \% \\
(73.97 \%-95.98 \%)\end{array}$ \\
\hline hypoalbuminemia & 14 & 457 & $<0.01$ & $82.70 \%$ & 0.0443 & Random & $\begin{array}{c}77.92 \% \\
(66.00 \%-87.85 \%)\end{array}$ \\
\hline Elevated LDH & 10 & 478 & $<0.01$ & $98.00 \%$ & 0.3994 & Random & $\begin{array}{c}80.59 \% \\
(42.53 \%-99.73 \%)\end{array}$ \\
\hline Elevated IL-6 & 11 & 527 & $<0.01$ & $91.90 \%$ & 0.0814 & Random & $89.3 \%(75.3 \%-97.88 \%)$ \\
\hline $\begin{array}{l}\text { Pulmonary ground glass } \\
\text { change }\end{array}$ & 14 & 271 & $<0.01$ & $71.00 \%$ & 0.0358 & Random & $\begin{array}{c}24.69 \% \\
(19.74 \%-29.99 \%) \\
\end{array}$ \\
\hline \multicolumn{8}{|l|}{ Severe disease and mortality } \\
\hline PICU & 48 & 1949 & $<0.01$ & $83.40 \%$ & 0.0346 & Random & $\begin{array}{c}72.79 \% \\
(66.75 \%-78.44 \%)\end{array}$ \\
\hline Shock & 42 & 1804 & $<0.01$ & $85.30 \%$ & 0.0384 & Random & $\begin{array}{c}55.68 \% \\
(48.50 \%-62.74 \%)\end{array}$ \\
\hline Mortality & 45 & 2010 & $<0.01$ & $39.00 \%$ & 0.0039 & Fixed & $1.00 \%(0.61 \%-1.48 \%)$ \\
\hline \multicolumn{8}{|l|}{ Treatment } \\
\hline $\begin{array}{l}\text { Invasive mechanical } \\
\text { ventilation }\end{array}$ & 46 & 1565 & $<0.01$ & $85.45 \%$ & 0.0451 & Random & $22.68 \%(16.91 \% 29.02 \%)$ \\
\hline ECMO & 37 & 1078 & $<0.01$ & $54.90 \%$ & 0.0109 & Random & $0.48 \%(0.03 \%-1.42 \%)$ \\
\hline IVIG & 47 & 1959 & $<0.01$ & $86.00 \%$ & 0.0413 & Random & $\begin{array}{c}82.15 \%(76.53 \%- \\
87.14 \%)\end{array}$ \\
\hline Aspirin & 20 & 592 & $<0.01$ & $89.20 \%$ & 0.0888 & Random & $\begin{array}{c}67.97 \%(53.77 \%- \\
80.64 \%)\end{array}$ \\
\hline Glucocorticoid & 40 & 1869 & $<0.01$ & $86.90 \%$ & 0.0401 & Random & $\begin{array}{c}59.32 \%(52.02 \%- \\
66.43 \%)\end{array}$ \\
\hline Vasoactive agent & 23 & 955 & $<0.01$ & $83.90 \%$ & 0.0493 & Random & $\begin{array}{c}45.79 \%(42.62 \%- \\
48.97 \%)\end{array}$ \\
\hline Positive inotropic & 24 & 939 & $<0.01$ & $87.40 \%$ & 0.0493 & Random & $\begin{array}{c}58.99 \%(48.57 \%- \\
69.02 \%)\end{array}$ \\
\hline IL-1 receptor antagonist & 16 & 516 & $<0.01$ & $76.50 \%$ & 0.0293 & Random & $3.61 \%(0.68 \%-8.73 \%)$ \\
\hline IL-6 receptor antagonist & 18 & 518 & $<0.01$ & $91.30 \%$ & 0.1060 & Random & $10.9 \%(2.79 \%-23.42 \%)$ \\
\hline Infliximab & 14 & 538 & $<0.10$ & $59.80 \%$ & 0.013 & Random & $6.68 \%(3.03 \%-11.62 \%)$ \\
\hline Antibiotic & 19 & 528 & $<0.11$ & 81.9 & 0.0562 & Random & $\begin{array}{c}90.96 \%(82.29 \%- \\
96.92 \%)\end{array}$ \\
\hline
\end{tabular}

$\mathrm{CRP}$ = C-reactive protein; $\mathrm{ESR}=$ erythrocyte sedimentation rate; $\mathrm{PCT}=$ procalcitonin; FIB = fibrinogen; $\mathrm{LDH}=$ lactate dehydrogenase; $\mathrm{IL}-6$ = interleukin-6; $\mathrm{BNP}=\mathrm{B}$ type natriuretic peptide; $\mathrm{ECMO}=$ extracorporeal membrane oxygenation; IVIG = intravenous immunoglobulin.

TABLE 2: Results of subgroup analysis.

\begin{tabular}{|c|c|c|c|c|c|c|c|}
\hline \multirow{2}{*}{ Outcome indicators } & \multirow{2}{*}{ Number of included studies } & \multirow{2}{*}{ Sample size } & \multicolumn{3}{|c|}{ Heterogeneity } & \multirow{2}{*}{ Effect of model } & \multirow{2}{*}{$\begin{array}{c}\text { Meta-analysis results } \\
\text { R\% }(95 \% \mathrm{CI})\end{array}$} \\
\hline & & & $P$ values & $I^{2}$ & $t^{2}(\%)$ & & \\
\hline \multicolumn{8}{|c|}{ Gastrointestinal symptoms } \\
\hline US & 21 & 1208 & $<0.01$ & 72.80 & 0.015 & Random & $87.77 \%(85.86 \%-89.55 \%)$ \\
\hline Europe & 21 & 753 & $<0.01$ & 79.90 & 0.0325 & Random & $76.53 \%(73.44 \%-79.48 \%)$ \\
\hline Other countries & 9 & 165 & $<0.01$ & 61.60 & 0.0231 & Random & $70.54 \%(63.38 \%-77.23 \%)$ \\
\hline$N<50$ & 44 & 757 & $<0.03$ & 70.10 & 0.0345 & Random & $84.11 \%(78.68 \%-88.87 \%)$ \\
\hline$N \geq 50$ & 7 & 1369 & $<0.04$ & 94.30 & 0.0243 & Random & $78.46 \%(67.80 \%-87.46 \%)$ \\
\hline \multicolumn{8}{|l|}{ Respiratory symptoms } \\
\hline US & 17 & 1128 & $<0.01$ & 88.60 & 0.0393 & Random & $47.68 \%(36.53 \%-58.94 \%)$ \\
\hline Europe & 12 & 489 & $<0.01$ & 84.90 & 0.0503 & Random & $53.77 \%(38.615-68.59 \%)$ \\
\hline Other countries & 7 & 136 & $<0.01$ & 84.60 & 0.0354 & Random & $67.01 \%(44.77 \%-85.83 \%)$ \\
\hline $\mathrm{N}<50$ & 30 & 500 & $<0.01$ & 81.30 & 0.0667 & Random & $54.98 \%(44.15 \%-65.57 \%)$ \\
\hline$N \geq 50$ & 6 & 1253 & $<0.01$ & 94.40 & 0.0236 & Random & $50.63 \%(37.88 \%-63.33 \%)$ \\
\hline \multicolumn{8}{|l|}{ Lymphocytopenia } \\
\hline US & 13 & 1206 & $<0.01$ & 93.08 & 0.0729 & Random & $58.70 \%(41.92 \%-74.50 \%)$ \\
\hline Europe & 7 & 77 & $<0.01$ & 70.90 & 0.0629 & Random & $84.01 \%(63.93 \%-96.84 \%)$ \\
\hline Other countries & 8 & 134 & $<0.01$ & 40.70 & 0.0112 & Fixed & $48.96 \%(40.55 \%-57.40 \%)$ \\
\hline$N<50$ & 25 & 351 & $<0.01$ & 77.40 & 0.0627 & Random & $62.05 \%(50.28 \%-73.14 \%)$ \\
\hline$N \geq 50$ & 3 & 855 & $<0.01$ & 98.40 & 0.0718 & Random & $58.4 \%(28.61 \%-85.23 \%)$ \\
\hline
\end{tabular}


Study

Omitting Riphagen S

Omitting Whittaker E

Omitting Toubiana J

Omitting Pouletty M

Omitting Dufort EM

Omitting Blumfield E

Omitting Rogo $\mathrm{T}$

Omitting Grimaud $M$

Omitting Diorio C

Omitting Sadiq M

Omitting Heidemann SM

Omitting Jain $\mathrm{S}$

Omitting Cantor A

Omitting Dhanalakshmi K

Omitting Mamishi S

Omitting Ng KF

Omitting Chiotos K

Omitting Blondiaux E

Omitting Ramcharan $\mathrm{T}$

Omitting Capone CA

Omitting Miller J

Omitting Pino R

Omitting Kest $\mathrm{H}$

Omitting Prata-Barbosa A

Omitting Godfred-Cato $S$

Omitting Webb K

Omitting Rostad CA

Omitting Blumfield E

Omitting Torres JP

Omitting Cheung EW

Omitting Riollano-Cruz M

Omitting Pereira MFB

Omitting Carter MJ

Omitting Belhadjer Z

Omitting Pang J

Omitting Lishman J

Omitting Feldstein LR

Omitting Wolfler A

Omitting Deep A

Omitting Corwin DJ

Omitting Hameed S

Omitting Lee PY

Omitting Jonat B

Omitting Felsenstein S

Omitting Moraleda C

Omitting Valverde I

Omitting Grazioli S

Omitting Gruber CN

Omitting Dionne A

Omitting Clouser KN

Omitting Prieto

Fixed-effects model

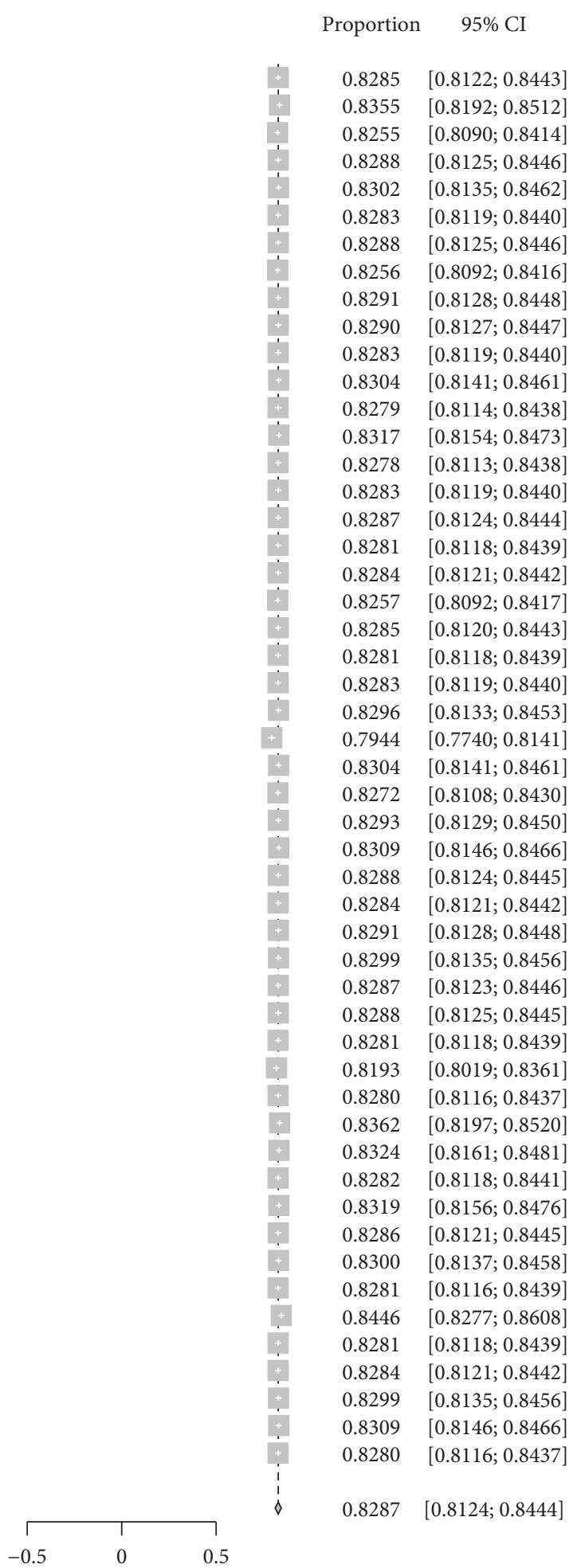

FIGURE 2: Sensitivity analysis of gastrointestinal symptoms. support. Similar to KD or toxic shock syndrome, most children had no obvious respiratory involvement. The incidence of respiratory symptoms in this study was only $53.02 \%$.

In early stages of the pandemic, this disease was considered to be KD caused by COVID-19, because there are many symptoms of $\mathrm{KD}$, such as rash, conjunctival congestion, chapped lips, and lymphadenitis. However, although there are some phenotypic similarities between MIS$\mathrm{C}$ and $\mathrm{KD}$, there are still many differences between the two diseases, such as the age of onset for $\mathrm{KD}$ being $<5$ years old, [68] and the median age in this study was $>5$ years old. In 


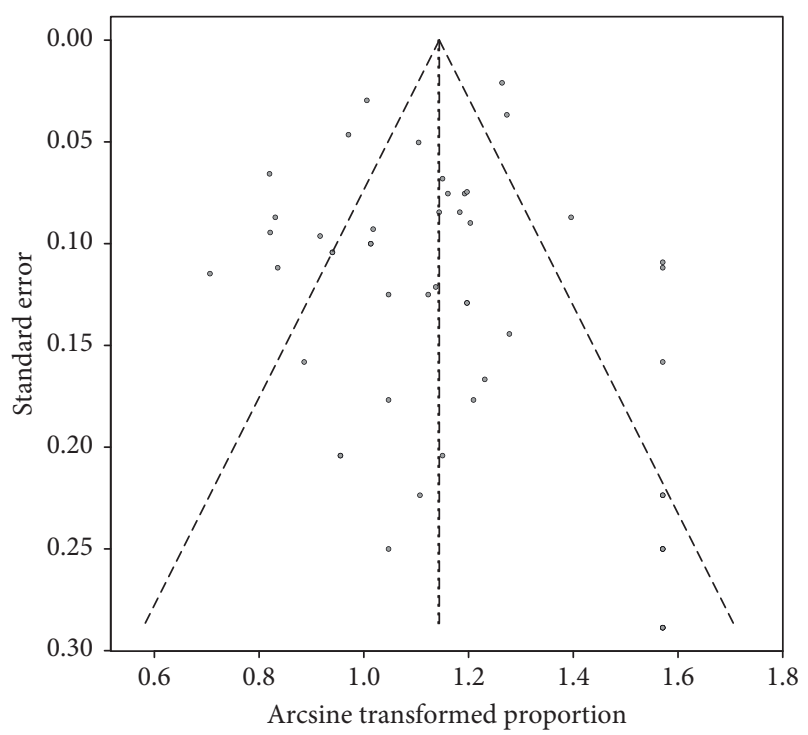

Figure 3: Funnel plot of publication bias of gastrointestinal symptoms.

$\mathrm{KD}$, platelet count generally increased, while thrombocytopenia accounted for $26.42 \%$ of MIS-C cases. Gastrointestinal symptoms in $\mathrm{KD}$ and the degree of myocardial dysfunction are also rare, while myocarditis, coronary artery dilatation, cardiac systolic function affects, and gastrointestinal symptoms accounted for $35.97 \%, 17.83 \%$, 56.32\%, and $82.72 \%$ of cases, respectively, in MIS-C.

A similar systematic review was published in August this year. The gastrointestinal tract of MIS-C patients [69] was similar to that of this study, and the gastrointestinal symptoms were often abdominal pain. When exploratory abdominal surgery was performed under MIS-C, gastrointestinal investigations also showed mesenteric lymphadenitis and serous effusions (ascites) in severe cases, which implied an active inflammatory reaction had occurred in the digestive system [70]. However, it has been proven that angiotensin-converting enzyme 2 is highly expressed in the small intestine, especially in the proximal and distal intestinal epithelial cells, so the small intestine is more vulnerable to SARS-CoV-2 infection [71].

Laboratory examinations of MIS-C showed a significant increase in various inflammatory indicators, such as CRP, ESR, FIB, ferritin, and LDH. It is worth noting that $89.3 \%$ of patients had elevated IL-6, which was as sensitive as $98.5 \%$ of patients with elevated CRP. However, lymphocyte reduction was noted in $51.51 \%$ of patients, which was much higher than in the majority of cases of COVID-19 in children [72].

Generally, MIS-C is a more systemic disease, increasing the likelihood of organ damage or impairments of liver, kidney, and/or heart function (rather than damaging the respiratory tract or facilitating the pneumonia infection process), which can easily lead to hypotension and circulatory failure. The rate of admission to the PICU was $72.79 \%$, and the rate of shock was $55.68 \%$.

Nevertheless, prognosis is good if appropriate treatment measures are taken as early as possible, such as treatment in the intensive care unit, close monitoring, intravenous injection of immunoglobulin $(82.15 \%$ utilization as used in the main treatment for $\mathrm{KD}$ ) and corticosteroids (59.32\% utilization), and use of biological agents and anticoagulants under the conditions recommended by appropriate specialists. Although the incidence of severe disease is high, $22.68 \%$ of the patients needed invasive mechanical ventilation and few patients $(0.48 \%)$ required extracorporeal membrane oxygenation, only $1.00 \%$ of pediatric patients die, so the overall outcomes are good; however, the long-term cardiovascular outcomes have not been determined.

The limitations of this study are as follows: (1) as the syndrome was known by multiple names at the start of the pandemic, the standards of research inclusion vary from country to country, which may lead to inclusion biases; (2) we found there is great heterogeneity among the studies, and there is significant publishing bias among several subgroups; and (3) this study was analyzed during an ongoing pandemic, and many areas affected by COVID-19 have not yet published clinical datasets, which may lead to inaccurate analysis results.

\section{Conclusions}

The incidence of MIS-C, which presents as multiple organ injuries and systemic inflammatory reactions, is low. MIS-C has a high rate of severity and patients are prone to symptoms of shock; however, if it is identified and treated in time, the mortality rate can remain low.

\section{Abbreviations \\ CRP: C-reactive protein \\ ESR: Erythrocyte sedimentation rate \\ PCT: Procalcitonin \\ FIB: Fibrinogen \\ LDH: Lactate dehydrogenase \\ IL-6: Interleukin-6 \\ BNP: B-type natriuretic peptide \\ ECMO: Extracorporeal membrane oxygenation \\ IVIG: Intravenous immunoglobulin.}

\section{Data Availability}

The data used to support the findings of this study are included within the article.

\section{Conflicts of Interest}

The authors declare that they have no conflicts of interest regarding the publication of this paper.

\section{Authors' Contributions}

Concept and design were proposed by Ji-Gan Wang and Zhi-Juan Zhong. Acquisition, analysis, and interpretation of data were carried out by Ji-Gan Wang, Yu-Heng Su, and YuLi Huang. Drafting of the manuscript was performed by JiGan Wang, Jun Fu, and Meng Li. Statistical analysis was conducted by Ji-Gan Wang and You-Min Ping. Administrative, technical, or material support was provided by Zi-Ji 
$\mathrm{Xu}$ and Hao Li. Supervision was done by Yan-Hao Chen and Yu-Li Huang. Ji-Gan Wang and Zhi-Juan Zhong contributed equally to this work.

\section{Acknowledgments}

This study was funded by Self-Funded Scientific Research Project of Guangxi Zhuang Autonomous Region Health Commission (nos. Z20210123 and Z20180083).

\section{Supplementary Materials}

Supplementary Table 2. Literature quality score table. Supplementary Table 1. Characteristics of studies included in the meta-analysis. (Supplementary Materials)

\section{References}

[1] D. Cucinotta and M. Vanelli, "WHO declares COVID-19 a pandemic," Acta Bio-Medica: Atenei Parmensis, vol. 91, pp. 157-160, 2020.

[2] M.-Y. Zhou, X.-L. Xie, Y.-G. Peng et al., "From SARS to COVID-19: what we have learned about children infected with COVID-19," International Journal of Infectious Diseases, vol. 96, pp. 710-714, 2020.

[3] K.-L. Shen, Y.-H. Yang, et al.,R.-M. Jiang, "Updated diagnosis, treatment and prevention of COVID-19 in children: experts' consensus statement (condensed version of the second edition)," World Journal of Pediatrics, vol. 16, no. 3, pp. 232-239, 2020.

[4] S. Riphagen, X. Gomez, C. Gonzalez-Martinez, N. Wilkinson, and P. Theocharis, "Hyperinflammatory shock in children during COVID-19 pandemic," The Lancet, vol. 395, no. 10237, pp. 1607-1608, 2020.

[5] M. Dolhnikoff, J. Ferreira Ferranti, R. A. de Almeida Monteiro et al., "SARS-CoV-2 in cardiac tissue of a child with COVID19-related multisystem inflammatory syndrome," The Lancet Child \& Adolescent Health, vol. 4, no. 10, pp. 790-794, 2020.

[6] L. A. Henderson, S. W Canna, K. G Friedman et al., "American College of Rheumatology clinical guidance for pediatric patients with multisystem inflammatory syndrome in children (MIS-C) associated with SARS-CoV-2 and hyperinflammation in COVID-19. Version 2,"Arthritis \& Rheumatology (Hoboken, N.J.), vol. 73, 2020.

[7] D. Moher, A. Liberati, J. Tetzlaff, and D. G. Altman, "Preferred Reporting Items for Systematic Reviews and Meta-Analyses: the PRISMA statement," PLoS Medicine, vol. 6, no. 7, Article ID e1000097, 2009.

[8] H. Tam, T. El Tal, E. Go, and R. S. M. Yeung, "Pediatric inflammatory multisystem syndrome temporally associated with COVID-19: a spectrum of diseases with many names," Canadian Medical Association Journal, vol. 192, no. 38, pp. E1093-e1096, 2020.

[9] C. Abraham, M. P. Kelly, R. West, and S. Michie, "The UK National Institute for Health and Clinical Excellence public health guidance on behaviour change: a brief introduction," Psychology, Health \& Medicine, vol. 14, no. 1, pp. 1-8, 2009.

[10] R. DerSimonian and R. Kacker, "Random-effects model for meta-analysis of clinical trials: an update," Contemporary Clinical Trials, vol. 28, no. 2, pp. 105-114, 2007.

[11] E. Whittaker, A. Bamford, J. Kenny et al., "Clinical characteristics of 58 children with a pediatric inflammatory multisystem syndrome temporally associated with SARSCoV-2," Jama, vol. 324, no. 3, pp. 259-269, 2020.

[12] J. Toubiana, C. Poirault, A. Corsia et al., "Kawasaki-like multisystem inflammatory syndrome in children during the covid-19 pandemic in Paris, France: prospective observational study," Bmj, vol. 369, 2020.

[13] M. Pouletty, C. Borocco, N. Ouldali et al., "Paediatric multisystem inflammatory syndrome temporally associated with SARS-CoV-2 mimicking Kawasaki disease (Kawa-COVID19): a multicentre cohort," Annals of the Rheumatic Diseases, vol. 79, no. 8, pp. 999-1006, 2020.

[14] E. M. Dufort, E. H. Koumans, E. J. Chow et al., "Multisystem inflammatory syndrome in children in New York state," New England Journal of Medicine, vol. 383, no. 4, pp. 347-358, 2020.

[15] E. Blumfield and T. L. Levin, "COVID-19 in pediatric patients: a case series from the Bronx, NY," Pediatric Radiology, vol. 50, no. 10, pp. 1369-1374, 2020.

[16] T. Rogo, K. Mathur, and M. Purswani, "Systemic inflammation with cardiac involvement in pediatric patients with evidence of COVID-19 in a community hospital in the Bronx, New York," Journal of the Pediatric Infectious Diseases Society, vol. 9, no. 4, pp. 502-503, 2020.

[17] M. Grimaud, "Acute myocarditis and multisystem inflammatory emerging disease following SARS-CoV-2 infection in critically ill children," Annals of Intensive Care, vol. 10, 69 pages, 2020.

[18] C. Diorio, S. E Henrickson, L. A Vella et al., "Multisystem inflammatory syndrome in children and COVID-19 are distinct presentations of SARS-CoV-2," The Journal of Clinical Investigation, vol. 130, pp. 5967-5975, 2020.

[19] M. Sadiq, O. A. Aziz, U. Kazmi et al., "Multisystem inflammatory syndrome associated with COVID-19 in children in Pakistan," The Lancet Child \& Adolescent Health, vol. 4, no. 10, pp. e36-e37, 2020.

[20] S. M. Heidemann, B. Tilford, C. Bauerfeld et al., "Three cases of pediatric multisystem inflammatory syndrome associated with COVID-19 due to SARS-CoV-2," American Journal of Case Reports, vol. 21, Article ID e925779, 2020.

[21] S. Jain, "Multisystem inflammatory syndrome in children with COVID-19 in Mumbai, India," Indian Pediatrics, vol. 57, pp. 1015-1019, 2020.

[22] A. Cantor, J. Miller, P. Zachariah, B. DaSilva, K. Margolis, and M. Martinez, "Acute hepatitis is a prominent presentation of the multisystem inflammatory syndrome in children: a singlecenter report," Hepatology, vol. 72, no. 5, pp. 1522-1527, 2020.

[23] K. Dhanalakshmi, "Epidemiological and clinical profile of pediatric inflammatory multisystem syndrome-temporally associated with SARS-CoV-2 (PIMS-TS) in Indian children," Indian Pediatrics, vol. 57, pp. 1010-1014, 2020.

[24] S. Mamishi, Z. Movahedi, M. Mohammadi et al., "Multisystem inflammatory syndrome associated with SARS-CoV-2 infection in 45 children: a first report from Iran," Epidemiology and Infection, vol. 148, e196 pages, 2020.

[25] K. F. Ng, T. Kothari, S. Bandi et al., "COVID-19 multisystem inflammatory syndrome in three teenagers with confirmed SARS-CoV-2 infection," Journal of Medical Virology, vol. 92, no. 11, pp. 2880-2886, 2020.

[26] K. Chiotos, H. Bassiri, E. M. Behrens et al., "Multisystem inflammatory syndrome in children during the coronavirus 2019 pandemic: a case series," Journal of the Pediatric Infectious Diseases Society, vol. 9, no. 3, pp. 393-398, 2020.

[27] E. Blondiaux, P. Parisot, A. Redheuil et al., "Cardiac MRI in children with multisystem inflammatory syndrome associated 
with COVID-19," Radiology, vol. 297, no. 3, pp. E283-e288, 2020.

[28] T. Ramcharan, O. Nolan, C. Y. Lai et al., "Paediatric inflammatory multisystem syndrome: temporally associated with SARS-CoV-2 (PIMS-TS): cardiac features, management and short-term outcomes at a UK tertiary paediatric hospital," Pediatric Cardiology, vol. 41, no. 7, pp. 1391-1401, 2020.

[29] C. A. Capone, A. Subramony, T. Sweberg et al., "Characteristics, cardiac involvement, and outcomes of multisystem inflammatory syndrome of childhood associated with severe acute respiratory syndrome coronavirus 2 infection," The Journal of Pediatrics, vol. 224, pp. 141-145, 2020.

[30] J. Miller, A. Cantor, P. Zachariah, D. Ahn, M. Martinez, and K. G. Margolis, "Gastrointestinal symptoms as a major presentation component of a novel multisystem inflammatory syndrome in children that is related to coronavirus disease 2019: a single center experience of 44 cases," Gastroenterology, vol. 159, no. 4, pp. 1571-1574, 2020.

[31] R. Pino, A. C. Izurieta, M. Ríos-Barnés et al., "Correspondence on: "Paediatric multisystem inflammatory syndrome temporally associated with SARS-CoV-2 mimicking Kawasaki disease (Kawa-COVID-19): a multicentre cohort" by Pouletty et al," Annals of the Rheumatic Diseases, vol. 2020, 2020.

[32] H. Kest, A. Kaushik, W. DeBruin, M. Colletti, and D. Goldberg, "Multisystem inflammatory syndrome in children (MIS-C) associated with 2019 novel coronavirus (SARSCoV-2) infection," Case Reports in Pediatrics, vol. 20204 pages, Article ID 8875987, 2020.

[33] A. Prata-Barbosa, F. Lima-Setta, G. R. D. Santos et al., "Pediatric patients with COVID-19 admitted to intensive care units in Brazil: a prospective multicenter study," Jornal de pediatria, vol. 96, no. 5, pp. 582-592, 2020.

[34] S. Godfred-Cato, B. Bryant, J. Leung et al., "COVID-19-associated multisystem inflammatory syndrome in childrenUnited States, March-July 2020," MMWR. Morbidity and Mortality Weekly Report, vol. 69, no. 32, pp. 1074-1080, 2020.

[35] K. Webb, D. R. Abraham, A. Faleye, M. McCulloch, H. Rabie, and C. Scott, "Multisystem inflammatory syndrome in children in South Africa," The Lancet Child \& Adolescent Health, vol. 4, no. 10, e38 pages, 2020.

[36] D. Matsubara, H. L. Kauffman, Y. Wang et al., "Echocardiographic findings in pediatric multisystem inflammatory syndrome associated with COVID-19 in the United States," Journal of the American College of Cardiology, vol. 76, no. 17, pp. 1947-1961, 2020.

[37] C. A. Rostad, A. Chahroudi, G. Mantus et al., "Quantitative SARS-CoV-2 serology in children with multisystem inflammatory syndrome (MIS-C)," Pediatrics, vol. 146, no. 6, 2020.

[38] E. Blumfield, T. L. Levin, J. Kurian, E. Y. Lee, and M. C. Liszewski, "Imaging findings in multisystem inflammatory syndrome in children (MIS-C) associated with coronavirus disease (COVID-19)," American Journal of Roentgenology, vol. 216, no. 2, pp. 507-517, 2021.

[39] J. P. Torres, G. Izquierdo, M. Acuña et al., "Multisystem inflammatory syndrome in children (MIS-C): report of the clinical and epidemiological characteristics of cases in Santiago de Chile during the SARS-CoV-2 pandemic," International Journal of Infectious Diseases, vol. 100, pp. 75-81, 2020.

[40] E. W. Cheung, P. Zachariah, M. Gorelik et al., "Multisystem inflammatory syndrome related to COVID-19 in previously healthy children and adolescents in New York City," Jama, vol. 324, no. 3, pp. 294-296, 2020.

[41] M. Riollano-Cruz, E. Akkoyun, E. Briceno-Brito et al., "Multisystem inflammatory syndrome in children related to
COVID-19: a New York City experience," Journal of Medical Virology, vol. 93, no. 1, pp. 424-433, 2020.

[42] M. F. B. Pereira, N Litvinov, S. C. L Farhat et al., "Severe clinical spectrum with high mortality in pediatric patients with COVID-19 and multisystem inflammatory syndrome," Clinics (Sao Paulo, Brazil), vol. 75, Article ID e2209, 2020.

[43] E. C. F. De Farias, J. Pedro Piva, M. L. F. M. F. De Mello et al., "Multisystem inflammatory syndrome associated with coronavirus disease in children," Pediatric Infectious Disease Journal, vol. 39, no. 11, pp. e374-e376, 2020.

[44] M. J. Carter, M. Fish, A. Jennings et al., "Peripheral immunophenotypes in children with multisystem inflammatory syndrome associated with SARS-CoV-2 infection," Nature Medicine, vol. 26, no. 11, pp. 1701-1707, 2020.

[45] Z. Belhadjer, M. Méot, F. Bajolle et al., "Acute heart failure in multisystem inflammatory syndrome in children in the context of global SARS-CoV-2 pandemic," Circulation, vol. 142, pp. 429-436, 2020.

[46] J. Pang, F. A. T. Boshier, N. Alders, G. Dixon, and J. Breuer, "SARS-CoV-2 polymorphisms and multisystem inflammatory syndrome in children," Pediatrics, vol. 146, no. 6, 2020.

[47] M. Perez-Toledo, S. E. Faustini, S. E. Jossi et al., "Serology confirms SARS-CoV-2 infection in PCR-negative children presenting with paediatric inflammatory multi-system syndrome," medRxiv: The Preprint Server for Health Sciences, 2020.

[48] J. Lishman, C. Kohler, C. de Vos et al., "Acute appendicitis in multisystem inflammatory syndrome in children with COVID-19," Pediatric Infectious Disease Journal, vol. 39, no. 12, pp. e472-e473, 2020.

[49] L. R. Feldstein, E. B. Rose, S. M. Horwitz et al., "Multisystem inflammatory syndrome in U.S. Children and adolescents," The New England Journal of Medicine, vol. 383, pp. 334-346, 2020.

[50] A. Wolfler, S. Mannarino, V. Giacomet, A. Camporesi, and G. Zuccotti, "Acute myocardial injury: a novel clinical pattern in children with COVID-19," The Lancet Child \& Adolescent Health, vol. 4, no. 8, pp. e26-e27, 2020.

[51] A. Deep, G. Upadhyay, P. du Pré et al., "Acute kidney injury in pediatric inflammatory multisystem syndrome temporally associated with severe acute respiratory syndrome coronavirus-2 pandemic: experience from PICUs across United Kingdom," Critical Care Medicine, vol. 48, no. 12, pp. 1809-1818, 2020.

[52] O. Y. Antúnez-Montes, M. I. Escamilla, A. F. Figueroa-Uribe et al., "COVID-19 and multisystem inflammatory syndrome in Latin American children," Pediatric Infectious Disease Journal, vol. 40, no. 1, pp. e1-e6, 2021.

[53] D. J. Corwin, L. F. Sartori, K. Chiotos et al., "Distinguishing multisystem inflammatory syndrome in children from Kawasaki disease and benign inflammatory illnesses in the SARS-CoV-2 pandemic," Pediatric Emergency Care, vol. 36, no. 11, pp. 554-558, 2020.

[54] S. Hameed, H. Elbaaly, C. E. L. Reid et al., "Spectrum of imaging findings at chest radiography, US, CT, and MRI in multisystem inflammatory syndrome in children associated with COVID-19," Radiology, vol. 298, no. 1, pp. E1-e10, 2021.

[55] P. Y. Lee, M. Day-Lewis, L. A. Henderson et al., "Distinct clinical and immunological features of SARS-CoV-2-induced multisystem inflammatory syndrome in children," Journal of Clinical Investigation, vol. 130, no. 11, pp. 5942-5950, 2020.

[56] B. Jonat, M. Gorelik, A. Boneparth et al., "Multisystem inflammatory syndrome in children associated with coronavirus disease 2019 in a children's hospital in New York City: patient 
characteristics and an institutional protocol for evaluation, management, and follow-up," Pediatric Critical Care Medicine, vol. 22, no. 3, pp. e178-e191, 2020.

[57] S. Felsenstein, E. Willis, H. Lythgoe et al., "Presentation, treatment response and short-term outcomes in paediatric multisystem inflammatory syndrome temporally associated with SARS-CoV-2 (PIMS-TS)," Journal of Clinical Medicine, vol. 9, no. 10, 3293 pages, 2020.

[58] C. Moraleda, "Multi-inflammatory syndrome in children related to SARS-CoV-2 in Spain," Clinical Infectious Diseases, vol. 72, 2020.

[59] I. Valverde, Y. Singh, J. Sanchez-de-Toledo et al., "Acute cardiovascular manifestations in 286 children with multisystem inflammatory syndrome associated with COVID-19 infection in Europe," Circulation, vol. 143, no. 1, pp. 21-32, 2021.

[60] S. Grazioli, F. Tavaglione, G. Torriani et al., "Immunological assessment of pediatric multisystem inflammatory syndrome related to coronavirus disease 2019," Journal of the Pediatric Infectious Diseases Society, vol. 142, 2020.

[61] C. N. Gruber, R. S. Patel, R. Trachtman et al., "Mapping systemic inflammation and antibody responses in multisystem inflammatory syndrome in children (MIS-C)," Cell, vol. 183, no. 4, pp. 982-995, 2020.

[62] A. Dionne, D. Y. Mah, M. B. F. Son et al., "Atrioventricular block in children with multisystem inflammatory syndrome," Pediatrics, vol. 146, no. 5, 2020.

[63] K. N. Clouser, J. Gadhavi, S. M. Bhavsar et al., "Short-term outcomes after multisystem inflammatory syndrome in children treatment," Journal of the Pediatric Infectious Diseases Society, vol. 10, no. 1, pp. 52-56, 2020.

[64] L. M. Prieto, B. Toral, A. LLorente, and D. Blázquez-Gamero, "Cardiovascular magnetic resonance imaging in children with pediatric inflammatory multisystem syndrome temporally associated with SARS-CoV-2 and heart dysfunction," Clinical Microbiology and Infection, vol. 27, no. 4, pp. 648-650, 2021.

[65] N. U. Falah, S. Hashmi, Z. Ahmed et al., "Kawasaki diseaselike features in 10 pediatric COVID-19 cases: a retrospective study," Cureus, vol. 12, 2020.

[66] M. S. Kelly, C. W. Valle, N. D. Fernandes, B. M. Cummings, M. Lahoud-Rahme, and J. S. Chiu, "Multisystem inflammatory syndrome in children: cardiac biomarker profiles and echocardiographic findings in the acute and recovery phases," Journal of the American Society of Echocardiography, vol. 33, no. 10, pp. 1288-1290, 2020.

[67] H. K. Kanthimathinathan and B. R. Scholefield, "Pediatric inflammatory multisystem syndrome: time to collaborate," Journal of the Pediatric Infectious Diseases Society, vol. 10, no. 3, pp. 227-229, 2020.

[68] B. W. McCrindle, A. H. Rowley, J. W. Newburger et al., "Diagnosis, treatment, and long-term management of Kawasaki disease: a scientific statement for health professionals from the American Heart Association," Circulation, vol. 135, no. 17, pp. e927-e999, 2017.

[69] J. Y. Abrams, S. E. Godfred-Cato, M. E. Oster et al., "Multisystem inflammatory syndrome in children associated with severe acute respiratory syndrome coronavirus 2: a systematic review," The Journal of Pediatrics, vol. 226, pp. 45-54, 2020.

[70] T.-H. Chen, W.-T. Kao, and Y.-H. Tseng, "Gastrointestinal involvements in children with COVID-related multisystem inflammatory syndrome," Gastroenterology, vol. 160, no. 5, pp. 1887-1888, 2021.
[71] W. Liang, Z. Feng, S. Rao et al., "Diarrhoea may be underestimated: a missing link in 2019 novel coronavirus," Gut, vol. 69, no. 6, pp. 1141-1143, 2020.

[72] M. A. Yoldas and H. Yoldas, "Pediatric COVID-19 disease: a review of the recent literature," Pediatric Annals, vol. 49, no. 7, pp. e319-e325, 2020. 